\title{
LOGARITHMIC BUNDLES AND LINE ARRANGEMENTS, AN APPROACH VIA THE STANDARD CONSTRUCTION
}

\author{
DANIELE FAENZI AND JEAN VALLÈS
}

\begin{abstract}
AвSTRACт. We propose an approach to study logarithmic sheaves $\mathscr{T}_{\mathbb{P}^{n}}\left(-\log D_{\mathscr{A}}\right)$ associated with hyperplane arrangements $\mathscr{A}$ on the projective space $\mathbb{P}^{n}$, based on projective duality, direct image functors and vector bundles methods. We focus on freeness of line arrangements having a point with high multiplicity.
\end{abstract}

\section{INTRODUCTION}

Let $\boldsymbol{k}$ be a field of characteristic zero, and let $\mathscr{A}=\left(H_{1}, \ldots, H_{m}\right)$ be a hyperplane arrangement in $\mathbb{P}^{n}=\mathbb{P}_{k}^{n}$, namely the $H_{i}$ 's are distinct hyperplanes of $\mathbb{P}^{n}$. The module of logarithmic derivations along the hyperplane arrangement divisor $D_{\mathscr{A}}=H_{1} \cup \cdots \cup H_{m}$, and its sheaf-theoretic counterpart $\mathscr{T}_{\mathbb{P}^{n}}\left(-\log D_{\mathscr{A}}\right)$ (Saito's sheaf of logarithmic vector fields) play a prominent role in the study of $\mathscr{A}$; let us only mention [Ter81, Sch00].

One main issue in the theory of arrangements is to what extent the sheaf $\mathscr{T}_{\mathbb{P}^{n}}\left(-\log D_{\mathscr{A}}\right)$ depends on the combinatorial type of $\mathscr{A}$, defined as the isomorphism type of the lattice $L_{\mathscr{A}}$ of intersections of hyperplanes in $\mathscr{A}$. This lattice is partially ordered by reverse inclusion, and is equipped with a rank function given by codimension (cf. [OT92]). An important conjecture of Terao (reported in [OT92]) asserts that if $\mathscr{A}$ and $\mathscr{A}^{\prime}$ have the same combinatorial type, and $\mathscr{T}_{\mathbb{P}^{n}}\left(-\log D_{\mathscr{A}}\right)$ splits as a direct sum of line bundles (i.e. $\mathscr{A}$ is free), the same should happen to $\mathscr{T}_{\mathbb{P}^{n}}\left(-\log D_{\mathscr{A}^{\prime}}\right)$.

In this paper we study the sheaf $\mathscr{T}_{\mathbb{P}^{n}}\left(-\log D_{\mathscr{A}}\right)$ relating it to the finite collection $Z$ of points in the dual space $\breve{P}^{n}$ associated with $\mathscr{A}$ (we write $\mathscr{A}=\mathscr{A}_{Z}$ when $Z=\left\{z_{1}, \ldots, z_{m}\right\}$ satisfies $H_{i}=H_{z_{i}}$ for all $i$, where $H_{z} \subset \mathbb{P}^{n}$ denotes the hyperplane corresponding to a point $\left.z \in \breve{\mathbb{P}}^{n}\right)$. Our first result is that $\mathscr{T}_{\mathbb{P}^{n}}\left(-\log D_{\mathscr{A}_{Z}}\right)$ is obtained via the so-called standard construction from the ideal sheaf $\mathscr{I}_{Z}(1)$, namely it is the direct image of the ideal sheaf $\mathscr{I}_{Z}(1)$ under the natural correspondence between $\mathbb{P}^{n}$ and $\breve{P}^{n}$ (Theorem 1).

On the projective plane, this allows to obtain special derivations (sections of $\mathscr{T}_{\mathbb{P}^{2}}\left(-\log D_{\mathscr{A}_{Z}}\right)$ ) from points of high multiplicity in $\mathscr{A}_{Z}$. Using this observation, we show that an arrangement $\mathscr{A}_{Z}$ of $2 k+r+1$ lines with a point of multiplicity between $k$ and $k+r+1$ is free with exponents $(k, k+r)$ if and only if $c_{2}\left(\mathscr{T}_{\mathbb{P}^{2}}\left(-\log D_{\mathscr{A}_{Z}}\right)\right)=k(k+r)$,

2010 Mathematics Subject Classification. 52C35, 14F05, 32S22.

Key words and phrases. Line arrangements, Freeness of arrangements, Terao's conjecture, Logarithmic sheaves, Projective duality.

Both authors partially supported by ANR-09-JCJC-0097-0 INTERLOW and ANR GEOLMI. 
see Theorem 2 . Here, by definition, $\mathscr{A}_{Z}$ free with exponents $(k, k+r)$ means that $\left.\mathscr{T}_{\mathbb{P}^{2}}\left(-\log D_{\mathscr{A}_{Z}}\right) \simeq \mathscr{O}_{\mathbb{P}^{2}}(-k) \oplus \mathscr{O}_{\mathbb{P}^{2}}(-r-k)\right)$, and we write Chern classes on $\mathbb{P}^{n}$ as integers, with obvious meaning. Note that the second Chern class is a very weak invariant of the combinatorial type of $\mathscr{A}_{Z}$. For real arrangements, one can push this criterion to points of slightly lower multiplicity, namely $k-1$ (in fact, a suitable technical assumption is needed, see Theorem 4 for the precise statement).

Next, we use the blow-up of the dual plane $\check{\mathbb{P}}^{2}$ to show that the splitting of $\mathscr{T}_{\mathbb{P}^{2}}\left(-\log D_{\mathscr{A}_{Z}}\right)$ on a general line of $\mathbb{P}^{2}$ is determined by the number $d_{Z}$, defined as the minimal integer $d$ such that there exists a curve of degree $d+1$ in the dual plane $\check{\mathbb{P}}^{2}$ passing through $Z$ and having multiplicity $d$ at a general point of $\breve{\mathbb{P}}^{2}$ (Theorem 3 ).

Using this approach, and the relations between the behavior of the arrangement obtained removing a line $H_{y}$ from $\mathscr{A}_{Z}$ and the possible order of trisecant lines to $Z$ in $\breve{\mathbb{P}}^{2}$ passing through $y$, we are lead to show that freeness is a combinatorial property for up to 12 lines (Theorem 5 ).

The paper is organised as follows. In the next section we set up the main correspondence between ideal sheaves of points in $\check{\mathbb{P}}^{n}$ and the sheaf of logarithmic derivations on $\mathbb{P}^{n}$. Section 2 contains our result on line arrangements having a point of high multiplicity. In Section 3 we show how to relate the number $d_{Z}$ and the generic splitting of the sheaf of logarithmic derivations of $\mathscr{A}_{Z}$. In Section 4 we develop the above mentioned refinement for real arrangements. In Section 5 we outline the relation of our method with the technique of deletion of one line from an arrangement, with a focus on freeness. Finally, Section 6 is devoted to arrangements of 12 or less lines in $\mathbb{P}^{2}$.

\section{DuAlity AND LOGARITHMiC VECTOR FIELDS}

Consider $\mathbb{P}^{n}=\mathbb{P}_{k}^{n}$, and let $Z=\left\{z_{1}, \ldots, z_{m}\right\}$ be a finite collection of points in the dual space $\breve{\mathbb{P}}^{n}$. Each point $y \in \breve{\mathbb{P}}^{n}$ corresponds to a hyperplane $H_{y}$ in $\mathbb{P}^{n}$ (and likewise we associate with $x \in \mathbb{P}^{n}$ a hyperplane of $\breve{P}^{n}$, denoted by $L_{x}$ ). So with $Z$ we can associate the hyperplane arrangement $\mathscr{A}_{Z}=\left(H_{z_{1}}, \ldots, H_{z_{m}}\right)$. The hyperplane arrangement divisor $D_{Z}=D_{\mathscr{A}_{Z}}$ is defined as $D_{Z}=\cup_{i=1}^{m} H_{z_{i}}$. Let $f_{i}$ be a linear form defining $H_{Z_{i}}$ and $f=\Pi_{i=1}^{m} f_{i}$ be an equation of $D_{Z}$.

Saito's sheaf of $\log$ arithmic vector fields $\mathscr{T}_{\mathbb{P}^{n}}\left(-\log D_{Z}\right)$ (see [Sai80]) is the sheafification of the module of logarithmic derivations associated to $D_{Z}$, mod out by the Euler derivation. It can be obtained as kernel of the map $\psi=\left(\partial_{0} f, \ldots, \partial_{n} f\right)$, where $x_{0}, \ldots, x_{n}$ are coordinates in $\mathbb{P}^{n}$, and we write $\partial_{i}=\partial / \partial\left(x_{i}\right)$. We will often abbreviate $\mathscr{T}_{Z}=\mathscr{T}_{\mathbb{P}^{n}}\left(-\log D_{Z}\right)$.

Our first result shows how to obtain $\mathscr{T}_{Z}$ from the ideal sheaf $\mathscr{I}_{Z}$ of $Z$ in $\breve{P}^{n}$ (we denote by $\mathscr{I}_{X / Y}$ the ideal sheaf of a subscheme $X$ of a scheme $Y$, and we suppress the notation $/ Y$ when it is clear from the context). Consider the flag variety:

$$
\mathbb{F}=\left\{(x, y) \in \mathbb{P}^{n} \times \check{\mathbb{P}}^{n} \mid x \in H_{y}\right\},
$$

and the projections $p$ and $q$ of $\mathbb{F}$ onto $\mathbb{P}^{n}$ and $\check{\mathbb{P}}^{n}$. It is well-known that $\mathbb{F} \simeq \mathbb{P}\left(\mathscr{T}_{\mathbb{P}^{n}}(-1)\right)$. 
Theorem 1. There is a natural isomorphism of sheaves of $\mathscr{O}_{\mathbb{P}^{n}}$-modules:

$$
\mathscr{T}_{Z} \simeq p_{*}\left(q^{*}\left(\mathscr{I}_{Z}(1)\right)\right) .
$$

Proof. This is somehow implicit in [FMV10, FV12]), still we give here a full proof. Let us consider the canonical exact sequence of coherent sheaves of $\mathscr{O}_{\breve{P} n}$-modules:

$$
0 \rightarrow \mathscr{I}_{Z}(1) \rightarrow \mathscr{O}_{\mathbb{P}^{n}}(1) \rightarrow \mathscr{O}_{Z}(1) \rightarrow 0
$$

Applying $p_{*} \circ q^{*}$ to this sequence, we get a long exact sequence:

$$
0 \rightarrow p_{*}\left(q^{*}\left(\mathscr{I}_{Z}(1)\right)\right) \rightarrow \mathscr{T}_{\mathbb{P}^{n}}(-1) \rightarrow p_{*}\left(q^{*}\left(\mathscr{O}_{Z}(1)\right)\right) \rightarrow \boldsymbol{R}^{1} p_{*}\left(q^{*}\left(\mathscr{I}_{Z}(1)\right)\right) \rightarrow 0 .
$$

Claim 1.1. The sheaf $\boldsymbol{R}^{1} p_{*}\left(q^{*}\left(\mathscr{I}_{Z}(1)\right)\right)$ is supported at the points $x \in \mathbb{P}^{n}$ such that $Z \cap L_{x}$ is not in general linear position.

To see this, first note that:

$$
\boldsymbol{R}^{i} p_{*}\left(q^{*}\left(\mathscr{I}_{Z}(1)\right)\right)=0, \text { for } i>1,
$$

which follows from the easy fact that $\mathrm{H}^{i}\left(L_{x}, \mathscr{I}_{Z \cap L_{x}}(1)\right)=0$ for all $i>1$ and all $x \in \mathbb{P}^{n}$. Then, by base change the support of the sheaf $\boldsymbol{R}^{1} p_{*}\left(q^{*}\left(\mathscr{I}_{Z}(1)\right)\right)$ is given by the points $x \in \mathbb{P}^{n}$ such that $\mathrm{H}^{1}\left(L_{x}, \mathscr{I}_{Z \cap L_{x}}(1)\right) \neq 0$, and this is non-zero if and only if $Z \cap L_{x}$ is not in general linear position.

Next, we observe that, for any $t \in \mathbb{Z}$, there is a natural isomorphisms:

$$
p_{*}\left(q^{*}\left(\mathscr{O}_{Z}(t)\right)\right) \simeq \bigoplus_{z \in Z} \mathscr{O}_{H_{z}} .
$$

To see this, first recall that $\mathscr{O}_{Z} \simeq \mathscr{O}_{Z}(t)$ for all $t$ since $Z$ has finite length. Further, $p_{*}\left(q^{*}\left(\mathscr{O}_{Z}(t)\right)\right)$ can be seen simply as $p_{*}\left(\mathscr{O}_{q^{-1}(Z)}\right)$ and since $q^{-1}(Z)$ is the disjoint union of the $\left\{H_{z} \mid x \in Z\right\}$, we get the desired isomorphism.

Let us now continue the proof of our theorem. Let $\mathscr{S}_{Z}$ be the singular locus of $D_{Z}$. This is defined by the vanishing of all partial derivatives of $f$, i.e. the generators of $\mathscr{I}_{\mathscr{S}_{Z} / \mathbb{P}^{n}}$ are given by the map $\psi$. Since $\mathscr{S}_{Z} \subset D_{Z}$, we have the natural exact sequence:

$$
0 \rightarrow \mathscr{O}_{\mathbb{P}^{n}}(-m) \stackrel{f}{\rightarrow} \mathscr{I}_{\mathscr{S}_{Z} / \mathbb{P}^{n}} \rightarrow \mathscr{I}_{\mathscr{S}_{Z} / D_{Z}} \rightarrow 0 .
$$

In view of the Euler relation, this sequence fits into a commutative diagram:

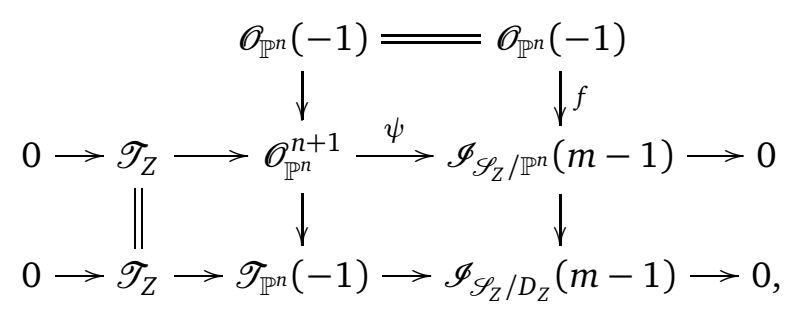

where the central column is the Euler sequence. From [Dol07, Proposition 2.4] desingularization gives an inclusion of $\mathscr{I}_{\mathscr{S}_{Z} / D_{Z}}(m)$ into $p_{*}\left(\omega_{q^{-1} Z} \otimes \omega_{\mathbb{P}^{n}}^{*}\right) \simeq p_{*}\left(q^{*}\left(\mathscr{O}_{Z}\right)\right)(1)$. 
So $\mathscr{T}_{Z}$ and $p_{*}\left(q^{*}\left(\mathscr{I}_{Z}(1)\right)\right)$ are both defined as kernel of maps $\mathscr{T}_{\mathbb{P}^{n}}(-1) \rightarrow p_{*}\left(q^{*}\left(\mathscr{O}_{Z}\right)\right)$, and we want to see that this turns them into isomorphic sheaves.

Claim 1.2. Any two sheaves defined as kernel of maps $\mathscr{T}_{\mathbb{P}^{n}}(-1) \rightarrow \bigoplus_{z \in Z} \mathscr{O}_{H_{z}}$ are isomorphic, provided that the first Chern class of both of them is $1-m$.

To see this, let $\alpha, \beta \in \operatorname{Hom}_{\mathscr{O}_{\mathbb{p}}}\left(\mathscr{T}_{\mathbb{P}^{n}}(-1), \bigoplus_{z \in Z} \mathscr{O}_{H_{z}}\right)$ set $E=\operatorname{ker}(\alpha), F=\operatorname{ker}(\beta)$, and assume $c_{1}(E)=c_{1}(F)=1-m$. For all $z \in Z$, we have $\operatorname{Hom}_{\mathscr{O}_{\mathbb{P}^{n}}}\left(\mathscr{T}_{\mathbb{P}^{n}}(-1), \mathscr{O}_{H_{z}}\right) \simeq$ $\mathrm{H}^{0}\left(\mathbb{P}^{n}, \Omega_{\mathbb{P}^{n}}(1) \otimes \mathscr{O}_{H_{z}}\right)$. Moreover, there is a natural isomorphism $\Omega_{\mathbb{P}^{n}}(1) \otimes \mathscr{O}_{H_{z}} \simeq \Omega_{H_{z}}(1) \oplus$ $\mathscr{O}_{H_{z}}$, So we have:

$$
\operatorname{Hom}_{\mathscr{O}_{\mathbb{P}^{n}}}\left(\mathscr{T}_{\mathbb{P}^{n}}(-1), \bigoplus_{z \in Z} \mathscr{O}_{H_{z}}\right) \simeq \bigoplus_{z \in Z} \mathrm{H}^{0}\left(H_{z}, \mathscr{O}_{H_{z}}\right),
$$

indeed $\mathrm{H}^{0}\left(H_{y}, \Omega_{H_{y}}(1)\right)=0$. Therefore, we may write $\alpha$ and $\beta$ as $\alpha=\left(\alpha_{z}\right)_{z \in Z}$ and $\beta=$ $\left(\beta_{z}\right)_{z \in Z}$ with $\alpha_{z}, \beta_{z} \in k$. The assumption $c_{1}(E)=1-m$ implies that $\alpha_{z} \neq 0$ for all $z \in Z$, and analogously $\beta_{z} \neq 0$ for all $z \in Z$ since $c_{1}(F)=1-m$. Now consider the automorphism $\gamma$ of $\bigoplus_{z \in Z} \mathscr{O}_{H_{z}}$ defined on each factor $\mathscr{O}_{H_{z}}$ as multiplication by $\frac{\beta_{z}}{\alpha_{z}}$. We have a commutative diagram:

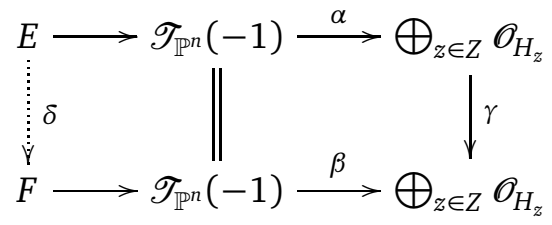

The map $\delta: E \rightarrow F$ induced by $\gamma$ has an inverse induced by $\gamma^{-1}$, and we get that $E$ is isomorphic to $F$.

To finish the proof, we only need to recall that $p_{*}\left(q^{*}\left(\mathscr{O}_{Z}\right)\right) \simeq \bigoplus_{z \in Z} \mathscr{O}_{H_{z}}$ by (2), and the first Chern class of both $\mathscr{T}_{Z}$ and $p_{*}\left(q^{*}\left(\mathscr{I}_{Z}(1)\right)\right)$ equals $1-m$ : for $\mathscr{T}_{Z}$ if follows from instance from (3), and for $p_{*}\left(q^{*}\left(\mathscr{I}_{Z}(1)\right)\right)$ it follows from (1), since $\boldsymbol{R}^{1} p_{*}\left(q^{*}\left(\mathscr{I}_{Z}(1)\right)\right)$ is supported in codimension at least 2 by Claim 1.1 , This finishes the proof of the theorem.

As an example of application of this description of $\mathscr{T}_{Z}$ as direct image, let us mention the following well-known result (a quick proof will be given in the next section).

Proposition 1.3. Let $\mathscr{A}$ be an arrangement of $m$ lines, $k \geq 0$ be an integer, $x$ be a point of multiplicity $k+1$ of $D_{\mathscr{A}}$. Set $\mathscr{A}^{\prime}=\mathscr{A} \backslash\{H \in \mathscr{A} \mid x \in H\}$. Then the following are equivalent:

i) the arrangement $\mathscr{A}$ is free with exponents $(k, m-k-1)$;

ii) any point of multiplicity $h \geq 2$ in $D_{\mathscr{A}^{\prime}}$ has multiplicity $h+1$ in $D_{\mathscr{A}}$.

\section{LINE ARRANGEMENTS WITH A POINT OF HIGH MULTIPLICITY}

Here we study freeness of line arrangement that admit a point having high multiplicity with respect to the exponents. Recall that a line arrangement $\mathscr{A}_{Z}$ is free with exponents $(a, b)$ if $\mathscr{T}_{Z} \simeq \mathscr{O}_{\mathbb{P}^{2}}(-a) \oplus \mathscr{O}_{\mathbb{P}^{2}}(-b)$. Of course, this implies that $c_{2}\left(\mathscr{T}_{Z}\right)=a b$. 
Theorem 2. Let $k \geq 1, r \geq 0$ be integers, set $m=2 k+r+1$, and consider a line arrangement $\mathscr{A}$ of $m$ lines with a point of multiplicity $h$ with $k \leq h \leq k+r+1$. Then $\mathscr{A}$ is free with exponents $(k, k+r)$ if and only if $c_{2}\left(\mathscr{T}_{\mathbb{P}^{2}}\left(-\log D_{\mathscr{A}}\right)\right)=k(k+r)$.

Remark 2.1. In the above setting, it turns out that if $h \geq k+r+2$, then $\mathscr{A}$ cannot be free with exponents $(k, k+r)$, see Corollary 3.2 .

Remark 2.2. A transparent way to compute the Chern class $c_{2}\left(\mathscr{T}_{\mathbb{P}^{2}}\left(-\log D_{\mathscr{A}}\right)\right)$ is the following. Set $b_{\mathscr{A}, h}$ for the number of points of multiplicity $h$ of $D_{\mathscr{A}}$ (we will also call them the points of multiplicity $h$ "of $\mathscr{A}$ "). Then we have the relations:

$$
\begin{aligned}
& b_{\mathscr{A}, 2}+3 b_{\mathscr{A}, 3}+6 b_{\mathscr{A}, 4}+\cdots=\left(\begin{array}{c}
m \\
2
\end{array}\right), \\
& b_{\mathscr{A}, 3}+3 b_{\mathscr{A}, 4}+6 b_{\mathscr{A}, 5}+\cdots=\left(\begin{array}{c}
m-1 \\
2
\end{array}\right)-c_{2}\left(\mathscr{T}_{\mathbb{P}^{n}}\left(-\log D_{\mathscr{A}}\right)\right) .
\end{aligned}
$$

The second equality, valid when not all lines in $\mathscr{A}$ pass through the same point, follows immediately from the long exact sequence:

$$
0 \rightarrow \mathscr{T}_{Z} \rightarrow \mathscr{O}_{\mathbb{P}^{n}}^{m-1} \rightarrow \mathscr{O}_{\mathbb{P}^{n}}^{m-3}(-1) \rightarrow \boldsymbol{R}^{1} p_{*}\left(q^{*}\left(\mathscr{I}_{Z}(1)\right)\right) \rightarrow 0,
$$

obtained by resolving $q^{*}\left(\mathscr{I}_{Z}(1)\right)$ in $\mathbb{P}^{2} \times \breve{\mathbb{P}}^{2}$ via:

$$
0 \rightarrow p r_{1}^{*}\left(\mathscr{O}_{\mathbb{P}^{2}}(-1)\right) \otimes p r_{2}^{*}\left(\mathscr{I}_{Z}(1)\right) \rightarrow p r_{2}^{*}\left(\mathscr{I}_{Z}(1)\right) \rightarrow q^{*}\left(\mathscr{I}_{Z}(1)\right) \rightarrow 0 .
$$

Here, $p r_{i}$ denote the projections onto the factors of $\mathbb{P}^{2} \times \breve{\mathbb{P}}^{2}$ (we refer to [FMV10] for more on the matrix of linear forms appearing in (6)). Indeed, in view of the proof of Claim 1.1 , the sheaf $\boldsymbol{R}^{1} p_{*}\left(q^{*}\left(\mathscr{I}_{Z}(1)\right)\right)$ is the direct sum of the $\mathscr{O}_{\left\langle x_{j}{ }^{m_{j}-2}\right\rangle}$, where the $x_{j}$ 's vary in the (set-theoretic) support of $\mathscr{S}_{Z}$ and $m_{j}=\operatorname{mult}\left(D_{\mathscr{A}_{Z}}, x_{j}\right)$. Here $\left\langle x^{i}\right\rangle$ denotes the $(i-1)^{\text {th }}$ infinitesimal neighborhood of $x$ in $\mathbb{P}^{2}$. This is the subscheme cut in $\mathbb{P}^{2}$ by the $i^{\text {th }}$ power of the ideal defining $x$. It has length $\left(\begin{array}{c}i+1 \\ 2\end{array}\right)$. Formula (5) thus follows computing Chern classes in (6).

Example 2.3. The result gives a quick way to show that an arrangement having the combinatorial type of the Hesse arrangement of the 12 lines passing through the 9 inflection points of a smooth complex plane cubic is free with exponents $(4,7)$.

To prove the theorem, we will need the following lemma. The way to use it will frequently be by contradiction: assume that a bundle $E$ with Chern classes as below does not split, hence take a non-zero element of $\mathrm{H}^{0}\left(\mathbb{P}^{2}, E(-1)\right)$, and look for a contradiction with some other property. We will sometimes call a non-zero element of $\mathrm{H}^{0}\left(\mathbb{P}^{2}, E(-1)\right)$ an unstable section.

Lemma 2.4. Let $E$ be a rank-2 vector bundle on $\mathbb{P}^{2}$ and assume $c_{1}(E)=-r$ for some $r \geq 0$ and $c_{2}(E)=0$. Then, the following are equivalent:

i) the bundle $E$ splits as $\mathscr{O}_{\mathbb{P}^{2}} \oplus \mathscr{O}_{\mathbb{P}^{2}}(-r)$, 


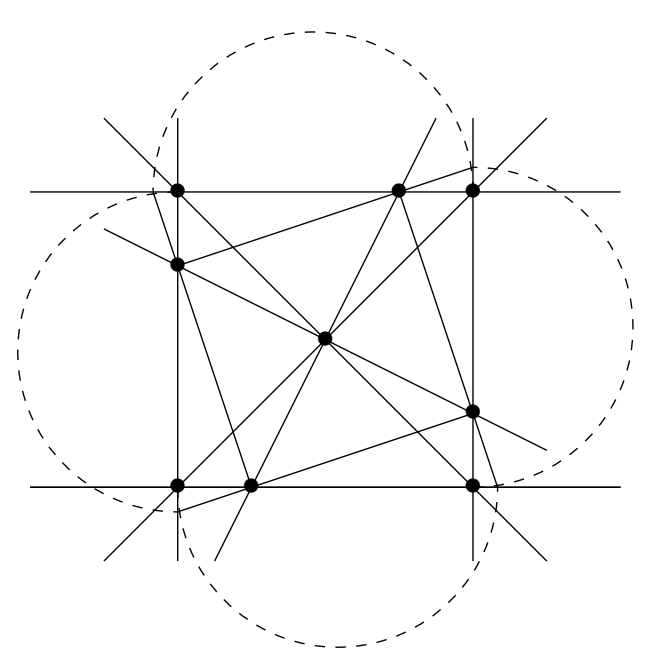

FigURE 1. Hesse arrangement

ii) we have $\mathrm{H}^{0}\left(\mathbb{P}^{2}, E(-1)\right)=0$,

iii) there is a line $H$ of $\mathbb{P}^{2}$ such that $E_{\mid H} \simeq \mathscr{O}_{H} \oplus \mathscr{O}_{H}(-r)$.

For any line $H$ of $\mathbb{P}^{2}$ we have $E_{\mid H} \simeq \mathscr{O}_{H}(s) \oplus \mathscr{O}_{H}(-r-s)$, for some integer $s \geq 0$.

Proof. Condition (ii) clearly implies (iii). The equivalence of (ii) and (iii) is proved in [EF80]. So it only remains to show that (iii) implies (ii), which we will now do.

Let $t$ be the smallest integer such that $\mathrm{H}^{0}\left(\mathbb{P}^{2}, E(t)\right) \neq 0$. By (iii) we know $t \geq 0$. Also, it is well-known that any non-zero global section $s$ of $E(t)$ vanishes along a subscheme $W$ of $\mathbb{P}^{2}$ of codimension $\geq 2$ and of length:

$$
c_{2}(E(t))=t(t-r) \geq 0
$$

We have an exact sequence:

$$
0 \rightarrow \mathscr{O}_{\mathbb{P}^{2}} \stackrel{s}{\rightarrow} E(t) \rightarrow \mathscr{I}_{W}(2 t-r) \rightarrow 0 .
$$

So $t=0$ would imply $X=\emptyset$ hence $\mathscr{I}_{W}(2 t-r) \simeq \mathscr{O}_{\mathbb{P}^{2}}(-r)$ and $E$ splits as $\mathscr{O}_{\mathbb{P}^{2}} \oplus \mathscr{O}_{\mathbb{P}^{2}}(-r)$ since $\operatorname{Ext}_{\mathbb{P}^{2}}^{1}\left(\mathscr{O}_{\mathbb{P}^{2}}(-r), \mathscr{O}_{\mathbb{P}^{2}}\right)=0$.

Then, it remains to rule out the case $t>0$. Hence, we assume $t>0$ i.e. $\mathrm{H}^{0}\left(\mathbb{P}^{2}, E\right)=$ 0 , and we look for a contradiction. By Riemann-Roch, the Euler characteristic $\chi(E)$ is positive, hence $\mathrm{H}^{2}\left(\mathbb{P}^{2}, E\right) \neq 0$, so $\mathrm{H}^{0}\left(\mathbb{P}^{2}, E(r-3)\right) \neq 0$ by Serre duality, indeed $E^{*} \simeq E(r)$. Therefore $t>0$ implies $t \leq r-3$. But by (7), $t>0$ implies $t \geq r$, a contradiction.

Let us now prove the last statement. Given a line $H$ of $\mathbb{P}^{2}$, we have $E_{\mid H} \simeq \mathscr{O}_{H}(s) \oplus$ $\mathscr{O}_{H}(-r-s)$ for some integer $s$, and we have to check that $s$ is non-negative. Let us assume $s<0$, and show that this leads to a contradiction. First, note that we may assume $s>-r$, for otherwise posing $s^{\prime}=-r-s$ we have $s^{\prime} \geq 0$ and we still have $E_{\mid H} \simeq \mathscr{O}_{H}\left(s^{\prime}\right) \oplus \mathscr{O}_{H}\left(-r-s^{\prime}\right)$.

Now, in case $-r<s<0$, we have an unstable section, namely $\mathrm{H}^{0}\left(\mathbb{P}^{2}, E(-1)\right) \neq 0$ since $E$ does not decompose as $\mathscr{O}_{\mathbb{P}^{2}} \oplus \mathscr{O}_{\mathbb{P}^{2}}(-r)$ (by the part we have already proved of this 
lemma). For all integers $t$, the exact sequence of restriction of $E(t)$ to $H$ reads:

$$
0 \rightarrow E(t-1) \rightarrow E(t) \rightarrow \mathscr{O}_{H}(t+s) \oplus \mathscr{O}_{H}(t-r-s) \rightarrow 0 .
$$

So $-r<s<0$ implies $\mathrm{H}^{0}\left(\mathbb{P}^{2}, E(t-1)\right) \simeq \mathrm{H}^{0}\left(\mathbb{P}^{2}, E(t)\right)$ for all $t \leq 0$, and this space is zero for $t \ll 0$. But this contradicts $\mathrm{H}^{0}\left(\mathbb{P}^{2}, E(-1)\right) \neq 0$.

We will now prove our theorem. We call a line $L \subset \check{\mathbb{P}}^{2}$ a $h$-secant line to $Z$ if $|L \cap Z| \geq h$. We add the adjective strict if we require equality. Recall that for all $h$, the number $b_{\mathscr{A}_{Z}, h}$ is the number of strict $h$-secants to $Z$.

Proof of Theorem 2 One direction if obvious. What we have to prove is that the condition on Chern classes is sufficient, so we assume $c_{2}\left(\mathscr{T}_{\mathbb{P}^{2}}\left(-\log D_{\mathscr{A}}\right)\right)=k(k+r)$. Let $Z$ be the set of $m$ points of $\breve{\mathbb{P}}^{2}$ corresponding to $\mathscr{A}$, so that $\mathscr{A}=\mathscr{A}_{Z}$ and $\mathscr{T}_{\mathbb{P}^{2}}\left(-\log D_{\mathscr{A}}\right)=\mathscr{T}_{Z}$. Since $\mathscr{A}$ has a point $x$ of multiplicity $h \geq k$, on the dual side there is a line $L=L_{x} \subset \breve{\mathbb{P}}^{2}$ that contains $h$ points of $Z$ (i.e. $L$ is a strict $h$-secant to $Z$ ), and leaves out the remaining $m-h$ points of $Z$. Set $Z^{\prime}=Z \backslash L$. Let $g$ be an equation of $L$ in $\breve{\mathbb{P}}^{2}$.

Restricting the ideal sheaf $\mathscr{I}_{Z}$ to $L$ we get the ideal sheaf of $h$ points in $\mathbb{P}^{1}$, i.e. $\mathscr{O}_{L}(-h)$. This gives an exact sequence:

$$
0 \rightarrow \mathscr{I}_{Z^{\prime}} \stackrel{g}{\rightarrow} \mathscr{I}_{Z}(1) \rightarrow \mathscr{O}_{L}(1-h) \rightarrow 0 .
$$

We apply $p_{*} \circ q^{*}$ to this exact sequence. It is easy to see that $p_{*}\left(q^{*}\left(\mathscr{I}_{Z^{\prime}}\right)\right)$ only depends on the length of $Z^{\prime}$ and is isomorphic to $\mathscr{O}_{\mathbb{P}^{2}}(h-m)$. Similarly, it is not hard to check (where in the second formula we replace the RHS by zero for $h \leq 2$ ):

$$
\begin{aligned}
& p_{*}\left(q^{*}\left(\mathscr{O}_{L}(1-h)\right)\right) \simeq \mathscr{O}_{\mathbb{P}^{2}}(1-h), \\
& \boldsymbol{R}^{1} p_{*}\left(q^{*}\left(\mathscr{O}_{L}(1-h)\right)\right) \simeq \mathscr{O}_{\left\langle x^{h-2}\right\rangle},
\end{aligned}
$$

(recall that $\left\langle x^{i}\right\rangle$ denotes the $(i-1)^{\text {th }}$ infinitesimal neighborhood of $x$ in $\mathbb{P}^{2}$ ).

Therefore $p_{*} \circ q^{*}$ of (8) gives:

$$
\begin{aligned}
0 & \rightarrow \mathscr{O}_{\mathbb{P}^{2}}(h-m) \rightarrow \mathscr{T}_{Z} \stackrel{\alpha}{\rightarrow} \mathscr{O}_{\mathbb{P}^{2}}(1-h) \rightarrow \\
& \rightarrow \boldsymbol{R}^{1} p_{*}\left(q^{*}\left(\mathscr{I}_{Z^{\prime}}\right)\right) \rightarrow \boldsymbol{R}^{1} p_{*}\left(q^{*}\left(\mathscr{I}_{Z}(1)\right)\right) \rightarrow \mathscr{O}_{\left\langle x^{h-2}\right\rangle} \rightarrow 0 .
\end{aligned}
$$

Now, an argument similar to Claim 1.1 shows that $\boldsymbol{R}^{1} p_{*}\left(q^{*}\left(\mathscr{I}_{Z^{\prime}}\right)\right)$ is supported at points $x$ such that $\mathrm{H}^{1}\left(L_{x}, \mathscr{I}_{Z^{\prime} \cap L_{x}}\right) \neq 0$, i.e. such that $\left|L_{x} \cap Z^{\prime}\right| \geq 2$ (bisecant lines to $\left.Z^{\prime}\right)$. The image of the map $\alpha$ above is then a sub-sheaf of $\mathscr{O}_{\mathbb{P}^{2}}(1-h)$, whose first Chern class is $1-h$ since all the sheaves in the second row of (9) are supported in codimension $\geq 2$. This means that $\operatorname{Im}(\alpha) \simeq \mathscr{I}_{\Gamma}(1-h)$, for some finite length subscheme $\Gamma \subset \mathbb{P}^{2}$, and we have:

$$
0 \rightarrow \mathscr{O}_{\mathbb{P}^{2}}(h-m) \rightarrow \mathscr{T}_{Z} \rightarrow \mathscr{I}_{\Gamma}(1-h) \rightarrow 0 .
$$

This subscheme parametrizes bisecant lines to $Z^{\prime}$ that meet $L$ away from $Z$. 
We apply now Lemma 2.4, If, by contradiction, the bundle $\mathscr{T}_{Z} \otimes \mathscr{O}_{\mathbb{P}^{2}}(k)$ did not split as $\mathscr{O}_{\mathbb{P}^{2}} \oplus \mathscr{O}_{\mathbb{P}^{2}}(-r)$, then we would have an unstable section, namely:

$$
\mathrm{H}^{0}\left(\mathbb{P}^{2}, \mathscr{T}_{Z} \otimes \mathscr{O}_{\mathbb{P}^{2}}(k-1)\right) \neq 0 .
$$

Note that the assumption $h \leq k+r+1=m-k$ gives $h+k-m-1<0$, so we have the vanishing $\mathrm{H}^{0}\left(\mathbb{P}^{2}, \mathscr{O}_{\mathbb{P}^{2}}(h+k-m-1)\right)=0$. So, from $(10)$, twisted by $\mathscr{O}_{\mathbb{P}^{2}}(k-1)$, we deduce:

$$
\mathrm{H}^{0}\left(\mathbb{P}^{2}, \mathscr{I}_{\Gamma}(k-h)\right) \neq 0,
$$

hence clearly $k \geq h$, which implies $h=k$ so $\mathrm{H}^{0}\left(\mathbb{P}^{2}, \mathscr{I}_{\Gamma}\right) \neq 0$. This says that $\Gamma$ is empty. But computing Chern classes in (10) twisted by $\mathscr{O}_{\mathbb{P}^{2}}(k-1)$ (and still with $h=k$ ) shows that $\Gamma$ has length $c_{2}\left(\mathscr{I}_{\Gamma}\right)=r+1$, a contradiction.

Proof of Proposition 1.3 Again, we let $Z$ be the set of $m$ points of $\breve{\mathbb{P}}^{2}$ corresponding to $\mathscr{A}$, so that $\mathscr{A}=\mathscr{A}_{Z}$ and $\mathscr{T}_{\mathbb{P}^{2}}\left(-\log D_{\mathscr{A}}\right)=\mathscr{T}_{Z}$. Since $\mathscr{A}$ has a point $x$ of multiplicity $k+1$, on the dual side there is a line $L=L_{x} \subset \breve{P}^{2}$ that contains $k+1$ points of $Z$ (i.e. $L$ is a strict $k+1$-secant to $Z$ ), and leaves out the remaining $m-k-1$ points of $Z$. Set $Z^{\prime}=Z \backslash L$. We have $\mathscr{A}^{\prime}=\mathscr{A}_{Z^{\prime}}$. We can then rewrite (9) as:

$$
\begin{aligned}
0 & \rightarrow \mathscr{O}_{\mathbb{P}^{2}}(k-m+1) \rightarrow \mathscr{T}_{Z} \rightarrow \mathscr{O}_{\mathbb{P}^{2}}(-k) \rightarrow \\
& \rightarrow \boldsymbol{R}^{1} p_{*}\left(q^{*}\left(\mathscr{I}_{Z^{\prime}}\right)\right) \rightarrow \boldsymbol{R}^{1} p_{*}\left(q^{*}\left(\mathscr{I}_{Z}(1)\right)\right) \rightarrow \mathscr{O}_{\left\langle x^{k-1}\right\rangle} \rightarrow 0 .
\end{aligned}
$$

Now, (iii) is equivalent to the fact that, for any $h \geq 2$, any strict $h$-secant to $Z^{\prime}$ is $(h+1)$ secant to $Z$. By the interpretation we gave in previous proof of the higher direct images appearing in (11), this is equivalent to exactness of the sequence:

$$
0 \rightarrow \boldsymbol{R}^{1} p_{*}\left(q^{*}\left(\mathscr{I}_{Z^{\prime}}\right)\right) \rightarrow \boldsymbol{R}^{1} p_{*}\left(q^{*}\left(\mathscr{I}_{Z}(1)\right)\right) \rightarrow \mathscr{O}_{\left\langle x^{k-1}\right\rangle} \rightarrow 0 .
$$

By (11), this is equivalent to exactness of:

$$
0 \rightarrow \mathscr{O}_{\mathbb{P}^{2}}(k-m+1) \rightarrow \mathscr{T}_{Z} \rightarrow \mathscr{O}_{\mathbb{P}^{2}}(-k) \rightarrow 0 .
$$

This is clearly equivalent to (i).

\section{BLOWING UP OF THE DUAL PLANE AND RESTRICTION TO LINES}

Let $H=H_{y}$ be a line in $\mathbb{P}^{2}$. Given a finite set of $m$ points $Z$ in $\check{\mathbb{P}}^{n}$, the associated sheaf $\mathscr{T}_{Z}$ restricts to $H_{y}$ as:

$$
\left(\mathscr{T}_{Z}\right)_{\mid H_{y}} \simeq \mathscr{O}_{H_{y}}\left(-a_{y}\right) \oplus \mathscr{O}_{H_{y}}\left(-b_{y}\right),
$$

for some integers $a_{y} \leq b_{y}$ with $a_{y}+b_{y}=m-1$. Let us work out the dual picture.

Definition 3.1. Let $y \in \breve{\mathbb{P}}^{2}$ and let $Z$ be a finite set of points of $\check{\mathbb{P}}^{2}$. We define $d_{Z, y}$ as the smallest positive integer $d$ such that there is a curve in $\breve{\mathbb{P}}^{2}$ of degree $d+1$ passing through $Z$ and having multiplicity $d$ at $y$. Equivalently, $d_{z, y}$ is the smallest integer $d$ such that:

$$
\mathrm{H}^{0}\left(\check{\mathbb{P}}^{2}, \mathscr{I}_{y}^{d} \otimes \mathscr{I}_{Z}(d+1)\right) \neq 0 .
$$


We also define $d_{z}$ as $\max _{y \in \breve{P}^{2}} d_{z, y}$.

Theorem 3. Let $Z$ be a finite set of points of $\check{\mathbb{P}}^{2}$ and $y \in \breve{\mathbb{P}}^{2} \backslash Z$. If $y$ lies on no trisecant line to $Z$, then $a_{y}=d_{Z, y}$.

Proof. We will first outline the application to our situation of the so-called standard construction, see [OSS80]. We consider the blow-up $\tilde{\mathbb{P}}$ of $\check{\mathbb{P}}^{2}$ at the point $y$, and we recall that $\tilde{\mathbb{P}}=p^{-1}\left(H_{y}\right)$, where $p$ is the projection map from the flag $\mathbb{F}$ to $\mathbb{P}^{2}$. Therefore we have an exact sequence:

$$
0 \rightarrow p^{*}\left(\mathscr{O}_{\mathbb{P}^{2}}(-1)\right) \rightarrow \mathscr{O}_{\mathbb{F}} \rightarrow \mathscr{O}_{\tilde{\mathbb{P}}} \rightarrow 0,
$$

with $\mathscr{O}_{\tilde{\mathbb{P}}} \simeq p^{*}\left(\mathscr{O}_{H_{y}}\right)$. We denote by $\tilde{p}$ and $\tilde{q}$ the induced projections from $\tilde{\mathbb{P}}$ to $H_{y}$ and to $\breve{\mathbb{P}}^{2}$. Tensoring the above exact sequence by $q^{*}\left(\mathscr{I}_{Z}(1)\right)$ and taking direct image by $p$ we get the long exact sequence:

$$
\begin{aligned}
0 & \rightarrow \mathscr{T}_{Z}(-1) \stackrel{f_{y}}{\rightarrow} \mathscr{T}_{Z} \rightarrow \tilde{p}_{*}\left(\tilde{q}^{*}\left(\mathscr{I}_{Z}(1)\right)\right) \rightarrow \\
& \rightarrow \boldsymbol{R}^{1} p_{*}\left(q^{*}\left(\mathscr{I}_{Z}(1)\right)\right) \stackrel{f_{y}}{\rightarrow} \boldsymbol{R}^{1} p_{*}\left(q^{*}\left(\mathscr{I}_{Z}(1)\right)\right) \rightarrow \boldsymbol{R}^{1} \tilde{p}_{*}\left(\tilde{q}^{*}\left(\mathscr{I}_{Z}(1)\right)\right) \rightarrow 0,
\end{aligned}
$$

where here $f_{y}$ is an equation of $H_{y}$ in $\mathbb{P}^{2}$. Note that, in the second row of the above diagram, all sheaves have finite length by Claim 1.1, and the kernel of the instance of $f_{y}$ in this row has the same length as its cokernel. Further note that, by base change over $x \in H_{y}$, we have:

$$
\boldsymbol{R}^{1} \tilde{p}_{*}\left(\tilde{q}^{*}\left(\mathscr{I}_{Z}(1)\right)\right) \otimes \boldsymbol{k}_{x} \simeq \mathrm{H}^{1}\left(L_{x}, \mathscr{I}_{Z \cap L_{x}}(1)\right),
$$

where $\boldsymbol{k}_{x}$ is the residue field at $x$. Therefore, looking at the line $L_{x}$ in $\check{\mathbb{P}}^{2}$ (which passes through $y$ ), the number of points in the intersection $L_{x} \cap Z$ equals $\operatorname{dim}\left(\boldsymbol{R}^{1} \tilde{p}_{*}\left(\tilde{q}^{*}\left(\mathscr{I}_{Z}(1)\right)\right) \otimes \boldsymbol{k}_{x}\right)+2$ (it is understood that if $\left|L_{x} \cap Z\right| \leq 2$ then this vector space is zero). Summing up, we have an exact commutative diagram:

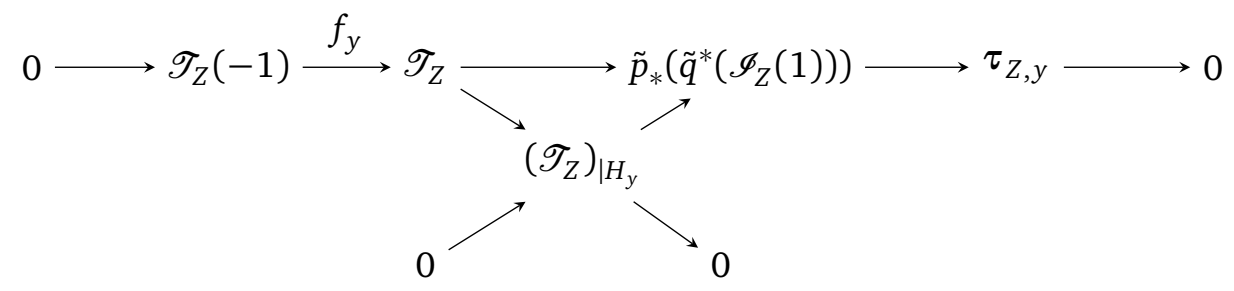

Here, the sheaf $\tau_{Z, y}$ has length $t_{Z, y}$. In particular, if $t_{Z, y}=0$ then:

$$
\tilde{p}_{*}\left(\tilde{q}^{*}\left(\mathscr{I}_{Z}(1)\right)\right) \simeq\left(\mathscr{T}_{Z}\right)_{\mid H_{y}} .
$$

Let us now head to the proof of our theorem. Set $a=a_{y}, b=b_{y}$. The decomposition $\left(\mathscr{T}_{Z}\right)_{\mid H_{y}} \simeq \mathscr{O}_{H_{y}}(-a) \oplus \mathscr{O}_{H_{y}}(-b)$ gives an injective map $\mathscr{O}_{H_{y}}(-a) \rightarrow \tilde{p}_{*}\left(\tilde{q}^{*}\left(\mathscr{I}_{Z}(1)\right)\right)$. Pulling back to $\tilde{\mathbb{P}}$, since $\tilde{p}^{*}\left(\mathscr{O}_{H_{y}}\right) \simeq \mathscr{O}_{\tilde{\mathbb{P}}}$, we get an injection:

$$
\mathscr{O}_{\tilde{\mathbb{P}}} \hookrightarrow \tilde{q}^{*}\left(\mathscr{I}_{Z}(1)\right) \otimes \tilde{p}^{*}\left(\mathscr{O}_{H_{y}}(a)\right) .
$$


Pushing down to $\check{\mathbb{P}}^{2}$, we get a map:

$$
\mathscr{O}_{\mathbb{P}^{2}} \hookrightarrow \mathscr{I}_{Z}(1) \otimes \tilde{q}_{*}\left(\tilde{p}^{*}\left(\mathscr{O}_{H_{y}}(a)\right)\right) \simeq \mathscr{I}_{Z}(1) \otimes \mathscr{I}_{y}^{a}(a) .
$$

Therefore we get $d_{Z, y} \geq a$. To check the opposite inequality, we show $\mathrm{H}^{0}\left(\check{\mathbb{P}}^{2}, \mathscr{I}_{y}^{a-1} \otimes \mathscr{I}_{Z}(a)\right)=0$. A non-zero element of this space, once pulled-back to $\check{\mathbb{P}}^{2}$, would give a map $\mathscr{O}_{\tilde{\mathbb{P}}^{2}} \hookrightarrow \mathscr{I}_{Z}(a)$, vanishing with multiplicity $a-1$ along the exceptional divisor $\tilde{q}^{-1}(y)$. By clearing $a-1$ times the equation of $\tilde{q}^{-1}(y)$, we get a map:

$$
\mathscr{O}_{\tilde{\mathbb{P}}} \hookrightarrow \tilde{q}^{*}\left(\mathscr{I}_{Z}(1)\right) \otimes \tilde{p}^{*}\left(\mathscr{O}_{H_{y}}(a-1)\right),
$$

hence, by pushing forward to $H_{y}$ via $\tilde{p}$, a map:

$$
\mathscr{O}_{H_{y}}(1-a) \hookrightarrow \tilde{p}_{*}\left(\tilde{q}^{*}\left(\mathscr{I}_{Z}(1)\right)\right) .
$$

This is incompatible with the splitting $\left(\mathscr{T}_{Z}\right)_{\mid H_{y}} \simeq \mathscr{O}_{H_{y}}(-a) \oplus \mathscr{O}_{H_{y}}(-b)$, with $a \leq b$.

Putting together the previous theorem and Lemma 2.4, we get the following result, somehow related to Yoshinaga's theorem, cf. [Yos04].

Corollary 3.2. Let $k \geq 1, r \geq 0$ be integers, set $m=2 k+r+1$, and consider a line arrangement $\mathscr{A}_{Z}$ associated with $m$ points $Z$ in $\check{\mathbb{P}}^{2}$ having $c_{2}\left(\mathscr{T}_{Z}\right)=k(k+r)$. Then the following are equivalent:

i) the arrangement $\mathscr{A}_{Z}$ is free with exponents $(k, k+r)$;

ii) there is a line $H=H_{y}$ in $\mathbb{P}^{2}$ such that $\left(\mathscr{T}_{Z}\right)_{\mid H_{y}} \simeq \mathscr{O}_{H_{y}}(-k) \oplus \mathscr{O}_{H_{y}}(-k-r)$;

iii) for a point $y \in \check{\mathbb{P}}^{2}$ lying in no trisecant line to $Z$, we have $d_{Z, y}=k$;

iv) we have $d_{Z}=k$.

In particular, if $Z$ has a $h$-secant line with $h \geq k+r+2$, then $\mathscr{A}_{Z}$ cannot be free.

Proof. The only two observations needed for the proof of the equivalence of the first four statements, besides Lemma 2.4 and Theorem 3, is the fact the maximal value $d_{Z}=k$ among the $d_{z, y}$ 's is attained at a generic point $y \in \check{\mathbb{P}}^{2}$. This in turn is clear by upper semicontinuity of the function $y \mapsto \operatorname{dim}_{k} \mathrm{H}^{0}\left(\check{\mathbb{P}}^{2}, \mathscr{I}_{y}^{d} \otimes \mathscr{I}_{Z}(d+1)\right)$.

For the last statement we argue as follows. Suppose that $Z$ has a strict $h$-secant line $L$ with $h \geq k+r+2$ and take a general point $y$ in $\breve{\mathbb{P}}^{2}$. Then we have a curve in $\breve{\mathbb{P}}^{2}$ of degree $m-h+1 \leq k$ through $Z$ and having multiplicity $m-h$ at $y$, namely the union of $L$ and of the $m-h$ lines joining $y$ with the $m-h$ points of $Z \backslash L$. Therefore $d_{Z, y} \leq k-1$ so $\mathscr{A}_{Z}$ is not free by the previous statements of this corollary.

\section{TWo DOUble POINTS ALIGNED WITH A POINT OF HIGH MULTIPLICITY}

For this section only, we consider real arrangements, namely we let $k=\mathbb{R}$. Let $k \geq 1$, $r \geq 0$ be integers, and set $m=2 k+r+1$. We consider here line arrangements $\mathscr{A}$ of $m$ lines having:

- a point $x_{0}$ of multiplicity $k-1$. 
- two points $x_{1}, x_{2}$, each of multiplicity at least 2 in $\mathscr{A}$, such that $\left\{x_{0}, x_{1}, x_{2}\right\}$ is contained in a line $H$ which is not in $\mathscr{A}$.

Theorem 4. Let $\mathscr{A}$ be as above. Then $\mathscr{A}$ is free with exponents $(k, k+r)$ if and only if $c_{2}\left(\mathscr{T}_{\mathbb{P}^{2}}\left(-\log D_{\mathscr{A}}\right)\right)=k(k+r)$.

Proof. Let $Z$ be the set of points in $\mathbb{P}^{2}$ corresponding to $\mathscr{A}$, so $\mathscr{A}=\mathscr{A}_{Z}$. We take a $(k-1)$-tuple point $x_{0}$ of $\mathscr{A}$, and let $H$ be the line in $\mathbb{P}^{2}$ containing $x_{0}, x_{1}, x_{2}$, with $x_{1}, x_{2}$ of multiplicity 2 or higher in $\mathscr{A}$. Again we let $L=L_{x}$ be the $(k-1)$-secant to $Z$ in $\check{\mathbb{P}}^{2}$, and set $Z^{\prime}=Z \backslash L$, this time $Z^{\prime}$ consists of $k+r+2$ points. We consider the exact sequence (8) (where this time $h=k-1$ ) and the long exact obtained by applying $p_{*} \circ q^{*}$ to it:

$$
\begin{aligned}
0 & \rightarrow \mathscr{O}_{\mathbb{P}^{2}}(-k-r-2) \rightarrow \mathscr{T}_{Z} \stackrel{\alpha}{\rightarrow} \mathscr{O}_{\mathbb{P}^{2}}(2-k) \rightarrow \\
& \rightarrow \boldsymbol{R}^{1} p_{*}\left(q^{*}\left(\mathscr{I}_{Z^{\prime}}\right)\right) \rightarrow \boldsymbol{R}^{1} p_{*}\left(q^{*}\left(\mathscr{I}_{Z}(1)\right)\right) \rightarrow \mathscr{O}_{\left\langle x^{k-3}\right\rangle} \rightarrow 0 .
\end{aligned}
$$

This time, the image of the map $\alpha$ above is $\mathscr{I}_{\Gamma}(2-k)$, where $\Gamma$ has length $2 r+2$, and the above sequence becomes:

$$
0 \rightarrow \mathscr{O}_{\mathbb{P}^{2}}(-k-r-2) \rightarrow \mathscr{T}_{Z} \rightarrow \mathscr{I}_{\Gamma}(2-k) \rightarrow 0 .
$$

Let us assume now that $\mathscr{T}_{Z}$ is not free, and show that this leads to a contradiction. First, by Lemma 2.4 we have $\mathrm{H}^{0}\left(\mathbb{P}^{2}, \mathscr{T}_{Z}(k-1)\right) \neq 0$, and we see from (12) that this gives $\mathrm{H}^{0}\left(\mathbb{P}^{2}, \mathscr{I}_{\Gamma}(1)\right) \neq 0$, therefore $\Gamma$ is contained in a line $H$ of $\mathbb{P}^{2}$. According to the interpretation we gave in the proof of Theorem 2 , the subscheme $\Gamma$ parametrizes (with multiplicity) the set of bisecant lines to $Z^{\prime}$ that meet $L$ away from $Z$. Since $x_{1}$ and $x_{2}$ give two such bisecants, we have that $H=H_{w}$. Note that by assumption $w$ does not lie in $Z$.

Summing up, we have the set $Z^{\prime}$ of $k+r+2$ points in $\check{\mathbb{P}}^{2}$, and a set $Z^{\prime \prime}$ of $k$ points in $L$, constituted by $w$ and $Z \cap L$, such that any bisecant line to $Z^{\prime}$ cuts $L$ along $Z^{\prime \prime}$. If we let now $L$ be the line at infinity in $\breve{\mathbb{P}}^{2}$, we see that $Z^{\prime}$ is a set of $k+r+2$ points of an affine 2-dimensional space, that determines at most $k$ directions. But, since we are working over $\mathbb{R}$, the set $Z^{\prime}$ should determine at least $k+r+1 \geq k+1$ directions, according to Ungar's theorem, see [Ung82]. This is a contradiction.

\section{SUB-ARRANGEMENT OBTAINED BY DELETION}

A classical and useful technique in the theory of arrangements consists in considering arrangements obtained from an arrangement $\mathscr{A}$ by adding a hyperplane out of $\mathscr{A}$, or deleting one of $\mathscr{A}$, or restricting $\mathscr{A}$ to a hyperplane of $\mathscr{A}$ (see [OT92] for a comprehensive treatment). Here we provide a different approach to this technique and outline some considerations on freeness of line arrangements based on our approach. Most of the results contained in this section are certainly known to experts, and can be proved with the classical techniques of deletion.

5.1. Deletion of a point and triple points along a hyperplane. Let $Z$ be a finite set of points in $\breve{\mathbb{P}}^{n}$ and let $z \in Z$. Set $Z^{\prime}=Z \backslash\{z\}$. We say that $\mathscr{A}_{Z^{\prime}}$ is a sub-arrangement of $\mathscr{A}_{Z}$, 
obtained by deletion of $z$. We have the exact sequence:

$$
0 \rightarrow \mathscr{I}_{Z} \rightarrow \mathscr{I}_{Z^{\prime}} \rightarrow \mathscr{O}_{Z} \rightarrow 0
$$

Applying $p_{*} \circ q^{*}$ to this sequence, we get:

$$
0 \rightarrow \mathscr{T}_{Z} \rightarrow \mathscr{T}_{Z^{\prime}} \stackrel{\beta_{0}}{\rightarrow} \mathscr{O}_{H_{z}} \stackrel{\beta_{1}}{\rightarrow} \boldsymbol{R}^{1} p_{*}\left(q^{*}\left(\mathscr{I}_{Z}(1)\right)\right) \stackrel{\beta_{2}}{\rightarrow} \boldsymbol{R}^{1} p_{*}\left(q^{*}\left(\mathscr{I}_{Z^{\prime}}(1)\right)\right) \rightarrow 0 .
$$

The kernel of the map $\beta_{1}$ above is a sub-sheaf of $\mathscr{O}_{H_{z}}$, which we refer to as the ideal of triple points of $\mathscr{A}_{Z}$ along $H_{z}$. For a point $y \in \check{\mathbb{P}}^{2}$, we write:

$$
t_{Z, y}=\sum_{x \in H_{y} \cap \mathscr{S}_{Z}}\left(\operatorname{mult}\left(D_{\mathscr{A}_{Z}}, x\right)-2\right) \text {. }
$$

Proposition 5.1. We have a short exact sequence:

$$
0 \rightarrow \mathscr{T}_{Z} \rightarrow \mathscr{T}_{Z^{\prime}} \rightarrow \mathscr{O}_{H_{z}}\left(-t_{Z, Z}\right) \rightarrow 0
$$

Proof. Given a point $x$ in $\mathbb{P}^{2}$, we denote again by $\left\langle x^{i}\right\rangle$ the $(i-1)^{\text {th }}$ infinitesimal neighborhood of $x$ in $\mathbb{P}^{2}$.

We have said that the sheaf $\boldsymbol{R}^{1} p_{*}\left(q^{*}\left(\mathscr{I}_{Z}(1)\right)\right)$ is the direct sum of the $\mathscr{O}_{\left\langle x_{j}{ }^{m_{j}-2}\right\rangle}$, where the $x_{j}$ 's vary in the support of $\mathscr{S}_{Z}$ and $m_{j}=\operatorname{mult}\left(D_{\mathscr{A}_{Z}}, x_{j}\right)$. Therefore, the kernel of the map $\beta_{2}$ above describes the difference between triple points of $\mathscr{A}_{Z}$ and triple points of $\mathscr{A}_{Z^{\prime}}$ each counted with multiplicity. By computing multiplicities, we get that the length of the support of $\operatorname{ker}\left(\beta_{2}\right)$ is precisely $t_{Z, z}$. Since $\operatorname{ker}\left(\beta_{2}\right)=\operatorname{Im}\left(\beta_{1}\right)$ has length $t_{Z, z}$, we get that $\operatorname{ker}\left(\beta_{1}\right)=\operatorname{Im}\left(\beta_{0}\right)$ has degree $-t_{Z, z}$, hence it is just $\mathscr{O}_{H_{z}}\left(-t_{Z, z}\right)$.

5.2. Some properties of freeness of line arrangements related to deletion. Here we give some simple relations between freeness of a given arrangements $\mathscr{A}_{Z}$ and the numbers $t_{Z, z}$, for $z \in Z$. Throughout the subsection, we let $k \geq 1, r \geq 0$ be integers, set $m=2 k+$ $r+1$, and we consider a set $Z$ of $m$ points of $\mathbb{P}^{2}$ and the corresponding line arrangement $\mathscr{A}_{Z}$.

Proposition 5.2. Assume $\mathscr{A}_{Z}$ is free with exponents $(k, k+r)$, let $z \in Z$ and set $Z^{\prime}=Z \backslash\{z\}$. Then, one of the following alternatives takes place:

i) $t_{Z, z}=k-1$ and $\mathscr{A}_{Z^{\prime}}$ is free with exponents $(k-1, k+r)$;

ii) $t_{Z, Z}=k+r-1$ and $\mathscr{A}_{Z^{\prime}}$ is free with exponents $(k, k+r-1)$;

iii) $t_{Z, z} \geq k+r$ and $\mathscr{A}_{Z^{\prime}}$ is not free.

Proof. Dualizing the exact sequence (13) (i.e., applying to it the functor $\mathscr{H}_{0} \mathrm{~m}_{\mathscr{O}_{\mathbb{P} 2}^{2}}\left(-, \mathscr{O}_{\mathbb{P}^{2}}\right)$ ), using the fact that $\mathscr{E} x t_{\mathscr{O}^{2}}^{1}\left(\mathscr{O}_{H_{Z}}(-t), \mathscr{O}_{\mathbb{P}^{2}}\right) \simeq \mathscr{O}_{H_{z}}(t+1)$ for all integer $t$, we obtain an exact sequence:

$$
0 \rightarrow \mathscr{T}_{Z^{\prime}}^{*} \rightarrow \mathscr{T}_{Z}^{*} \rightarrow \mathscr{O}_{H_{z}}\left(t_{Z, z}+1\right) \rightarrow 0
$$


Here $(-)^{*}$ denotes the dual of a vector bundle. Since $\mathscr{T}_{Z}^{*} \simeq \mathscr{O}_{\mathbb{P}^{2}}(k) \oplus \mathscr{O}_{\mathbb{P}^{2}}(k+r)$, we have thus a a surjective map:

$$
\mathscr{O}_{\mathbb{P}^{2}}(k) \oplus \mathscr{O}_{\mathbb{P}^{2}}(k+r) \rightarrow \mathscr{O}_{H_{z}}\left(t_{Z, z}+1\right) .
$$

Then, it is clear that $t_{Z, z} \geq k-1$ for otherwise there could not be an epimorphism as above. Also, it is clear that in case (ii) the kernel bundle of the above map splits in the desired way, since the map above factors as:

$$
\mathscr{T}_{Z}^{*} \rightarrow \mathscr{O}_{\mathbb{P}^{2}}(k) \rightarrow \mathscr{O}_{H_{z}}(k),
$$

where the first map is the projection onto the direct summand $\mathscr{O}_{\mathbb{P}^{2}}(k)$ and the second map is the canonical surjection. The case (iii) is analogous.

Let us prove now the case (iii). We consider again the exact sequence (14). We twist it by $-t_{Z, z}-1$ and take the long exact sequence of cohomology. Since $t_{Z, z} \geq k+r$ we get $\mathrm{H}^{1}\left(\mathbb{P}^{2}, \mathscr{T}_{Z^{\prime}}^{*}\left(-t_{Z, z}-1\right)\right) \neq 0$ which proves that $\mathscr{T}_{Z^{\prime}}$ does not decompose as a direct sum of line bundles.

In the same spirit, we have the following proposition.

Proposition 5.3. Assume $c_{2}\left(\mathscr{T}_{Z}\right)=k(k+r)$. Then:

i) for all $z \in Z$, we have $\left.t_{Z, z} \notin\right] k-1, k+r-1[$;

ii) if there is $z \in Z$ such that $t_{Z, z}=k-1$ or $t_{Z, z}=k+r-1$, then $\mathscr{A}_{Z}$ is free with exponents $(k, k+r)$;

iii) if there is $z \in Z$ such that $t_{Z, z}<k-1$, then $\mathscr{A}_{Z}$ is not free.

Moreover, if $\mathscr{A}_{Z}$ is not free, but has the same combinatorial type of a free arrangement, then for all $z \in Z$ we have $t_{Z, z} \geq k+r$.

Proof. Consider again the exact sequence obtained in the proof of the previous proposition (from which we borrow the notation also):

$$
0 \rightarrow \mathscr{T}_{Z^{\prime}}^{*} \rightarrow \mathscr{T}_{Z}^{*} \rightarrow \mathscr{O}_{H_{z}}\left(t_{Z, z}+1\right) \rightarrow 0 .
$$

Consider now the restriction to the line $H_{z}$ of $\mathscr{T}_{Z}^{*}$. This splits as $\mathscr{O}_{H_{z}}(k-s) \oplus \mathscr{O}_{H_{z}}(k+r+s)$, for some integer $s \geq 0$ by Lemma 2.4 , indeed one computes $c_{2}\left(\mathscr{T}_{Z}(-k)\right)=0$. So we get an epimorphism:

$$
\mathscr{O}_{H_{z}}(k-s) \oplus \mathscr{O}_{H_{z}}(k+r+s) \rightarrow \mathscr{O}_{H_{z}}\left(t_{Z, z}+1\right) .
$$

Now, in case $t_{Z, Z}=k-1$ or $t_{Z, z}=k+r-1$, this forces $s=0$, hence $\mathscr{T}_{Z}$ is free by Corollary 3.2. This gives (iii). By the same corollary, since $t_{z, z}<k-1$ forces $s<0$, we get (iii). To see (ii), we note that an epimorphism of the form (15) cannot exist in this range.

To check the last statement, note that $\mathscr{A}_{Z}$ cannot have the combinatorial type of a free arrangement $\mathscr{A}_{Z_{0}}$ if $t_{Z, z}<k-1$, for necessarily we have $c_{2}\left(\mathscr{T}_{Z_{0}}\right)=k(k+r)$ and we would get $t_{Z_{0}, z_{0}}<k-1$ for some $z_{0} \in Z_{0}$ contradicting (iii). Also, we cannot have $t_{Z, z}=k-1$ or 
$t_{Z, z}=k+r-1$ for any $z \in Z$ for otherwise $\mathscr{A}_{Z}$ would be free by (iii). Then by (ii) we get $t_{Z, z} \geq k+r$ for all $z \in Z$.

\section{Combinatorial NATURE OF FREENESS FOR UP TO 12 Lines}

In this section we work over $\boldsymbol{k}=\mathbb{C}$. The aim here will be to show that Terao's conjecture holds for arrangements of up to 12 complex projective lines. As far as we know, this had been checked for up to 10 lines, see [WY07]. The reason for devoting a paragraph to such a little progress is that we still hope that our methods involving the unstable section of Section 2 can help treating more cases. Also, it seems to us the combinatorial subtleties are ruled out by Theorem 2 for less than 11 lines, so this seems to be the first intriguing case.

Theorem 5. Terao's conjecture holds for up to 12 lines in $\mathbb{P}_{\mathbb{C}}^{2}$.

We fix again our notation: we consider a finite set of point $Z$ in $\breve{\mathbb{P}}^{2}$ and the corresponding arrangement $\mathscr{A}=\mathscr{A}_{Z}$.

Lemma 6.1. Assume $\mathscr{A}$ is free with exponents $(k, k+r)$, with $r \geq 0$.

i) There is no h-secant line to $Z$, for $h>k+r+1$.

ii) Assume that $Z$ is non-degenerate and has no 4-secant line. Then $(k, k+r)$ take value $(1,1),(1,2),(2,2),(2,3),(3,3),(3,4)$ or $(4,4)$. In the last case, $\mathscr{A}$ has the combinatorial type of the arrangement (sometimes called "dual Hesse) given by the 9 lines corresponding to the inflection points of a smooth cubic curve in $\breve{\mathbb{P}}^{2}$.

Proof. The first claim follows by looking at the exact sequence (8). Indeed, let $L$ be a strict $h$-secant line to $Z$, with $h>k+r+1$. Then $Z^{\prime}=Z \backslash L$ consists of $2 k+r+1-h$ points, and we have an inclusion $\mathscr{I}_{Z^{\prime}} \rightarrow \mathscr{I}_{Z}(1)$. Applying $p_{*} \circ q^{*}$ to this inclusion we get a nonzero map:

$$
\mathscr{O}_{\mathbb{P}^{2}}(h-2 k-r-1) \rightarrow \mathscr{T}_{Z} \simeq \mathscr{O}_{\mathbb{P}^{2}}(-k) \oplus \mathscr{O}_{\mathbb{P}^{2}}(-k-r) .
$$

This is impossible since $h-2 k-r-1>-k$, and we get (ii).

Let us look at (iii). Since $Z$ is non-degenerate, we have $k \geq 1$ and by (ii) we get $k+r \leq 4$. The relations (4) and (5) yield:

$$
b_{\mathscr{A}, 2}=-k^{2}-k s-s^{2}+2 k-2 s .
$$

It is easy to see that this gives a negative number for $r \geq 2$, or for $r=1, k \geq 4$, and also for $r=0, k \geq 5$. This leaves out the desired cases only. In case $r=0, k=4$, we get $b_{\mathscr{A}, 3}=12$ and $b_{\mathscr{A}, 3}=0$, which is the combinatorial type of the arrangement of 9 lines dual to the 9 inflection points of a smooth cubic curve.

Proof of Theorem 5 The case when $Z$ is degenerate is trivial. Theorem 2 and the previous lemma only leave out the cases of line arrangements $\mathscr{A}$ of 11 lines with $c_{2}\left(\mathscr{T}_{\mathbb{P}^{n}}\left(-\log D_{\mathscr{A}}\right)\right)=25$ or of 12 lines with $c_{2}\left(\mathscr{T}_{\mathbb{P}^{n}}\left(-\log D_{\mathscr{A}}\right)\right)=30$, having in both cases a quadruple point and no quintuple points. 
We look at the first case, which in our notation has $k=5, r=0$; the second case is completely analogous, and in fact a bit easier, so we will only say a word about it at the end of the proof. We consider thus an arrangement $\mathscr{A}$ with $c_{2}\left(\mathscr{T}_{\mathbb{P}^{n}}\left(-\log D_{\mathscr{A}}\right)\right)=25$ and such that $\mathscr{T}_{\mathbb{P}^{n}}\left(-\log D_{\mathscr{A}}\right)$ is not free. Also, we can assume that there is a line arrangement $\mathscr{A}_{0}$, with the same combinatorial type of $\mathscr{A}$, such that $\mathscr{T}_{\mathbb{P}^{2}}\left(-\log D_{\mathscr{A}_{0}}\right)$ is free with exponents $(5,5)$.

Let $Z$ and $Z_{0}$ be the corresponding set of points in $\mathbb{P}^{2}$, so $\mathscr{A}=\mathscr{A}_{Z}, \mathscr{A}_{0}=\mathscr{A}_{Z_{0}}$, let $x$ be a quadruple point of $\mathscr{A}$, and set $L$ for the corresponding 4-secant line to $Z$ in $\check{\mathbb{P}}^{2}$. We are in the situation of Theorem 4, only without the assumptions of reality and of existence of $x_{1}, x_{2}$. However we let $Z^{\prime}=Z \backslash L$ (this time $Z^{\prime}$ consists of 7 points), and we consider the exact sequence (8) and the long exact obtained by applying $p_{*} \circ q^{*}$ :

$$
0 \rightarrow \mathscr{O}_{\mathbb{P}^{2}}(-7) \rightarrow \mathscr{T}_{Z} \stackrel{\alpha}{\rightarrow} \mathscr{O}_{\mathbb{P}^{2}}(-3) \rightarrow \boldsymbol{R}^{1} p_{*}\left(q^{*}\left(\mathscr{I}_{Z^{\prime}}\right)\right) \rightarrow \boldsymbol{R}^{1} p_{*}\left(q^{*}\left(\mathscr{I}_{Z}(1)\right)\right) \rightarrow \mathscr{O}_{\left\langle x^{2}\right\rangle} \rightarrow 0 .
$$

Again $\operatorname{Im}(\alpha)=\mathscr{I}_{\Gamma}(-3)$, where $\Gamma$ is a subscheme of $\mathbb{P}^{2}$ of length 4 , so that the above sequence becomes:

$$
0 \rightarrow \mathscr{O}_{\mathbb{P}^{2}}(-7) \rightarrow \mathscr{T}_{Z} \rightarrow \mathscr{I}_{\Gamma}(-3) \rightarrow 0 .
$$

Since $\mathscr{T}_{Z}$ is not free by assumption, by Lemma 2.4 we have $\mathrm{H}^{0}\left(\mathbb{P}^{2}, \mathscr{T}_{Z}(4)\right) \neq 0$, and again we get $\mathrm{H}^{0}\left(\mathbb{P}^{2}, \mathscr{I}_{\Gamma}(1)\right) \neq 0$ so that $\Gamma$ is contained in a line $H_{w}$, for some $w \in \check{\mathbb{P}}^{2}$.

Claim 6.2. The point $w$ does not lie in $Z$.

Proof of claim 6.2 Recall that, by the proof of Theorem 2 , the subscheme $\Gamma$ parametrizes (with multiplicity) the set of bisecant lines to $Z^{\prime}$ that meet $L$ away from $Z$. The fact that $\Gamma$ sits in $H_{w}$ means that these lines all meet at $w$.

If $w$ belongs to $Z$, then this is a combinatorial property that must also hold for $Z_{0}$, namely the subscheme $\Gamma_{0}$ associated to $Z_{0}$ should be contained in a line $H_{w_{0}}$ corresponding to the meeting point $w_{0}$. But $Z_{0}$ is free with exponents $(5,5)$, so by Lemma 2.4 we have $\mathrm{H}^{0}\left(\mathbb{P}^{2}, \mathscr{T}_{Z_{0}}(4)\right)=\mathrm{H}^{0}\left(\mathbb{P}^{2}, \mathscr{I}_{\Gamma_{0}}(1)\right)=0$. Hence $\Gamma_{0}$ lies in no line.

Claim 6.3. The point $w$ lies in $L$, and the extended arrangement $\tilde{Z}=Z \cup\{w\}$ is free with exponents $(4,7)$.

Proof of claim 6.3 Since $\Gamma$ has length 4 and lies in $H_{w}$, we get a projection $\mathscr{I}_{\Gamma} \rightarrow \mathscr{O}_{H_{w}}(-4)$, with kernel $\mathscr{O}_{\mathbb{P}^{2}}(-4)$, hence an exact sequence:

$$
0 \rightarrow \mathscr{O}_{\mathbb{P}^{2}}(-4) \rightarrow \mathscr{I}_{\Gamma}(-3) \rightarrow \mathscr{O}_{H_{w}}(-7) \rightarrow 0 .
$$

Composing the above projection with the epimorphism $\mathscr{T}_{Z} \rightarrow \mathscr{I}_{\Gamma}(-3)$ we get a surjective map $\mathscr{T}_{Z} \rightarrow \mathscr{O}_{H_{w}}(-7)$. This gives an exact sequence:

$$
0 \rightarrow \mathscr{O}_{\mathbb{P}^{2}}(-7) \oplus \mathscr{O}_{\mathbb{P}^{2}}(-4) \rightarrow \mathscr{T}_{Z} \rightarrow \mathscr{O}_{H_{w}}(-7) \rightarrow 0,
$$

obtained by observing that the map $\mathscr{O}_{\mathbb{P}^{2}}(-7) \rightarrow \mathscr{T}_{Z}$ does not factor through $\mathscr{O}_{\mathbb{P}^{2}}(-4) \rightarrow \mathscr{T}_{Z}$ (indeed, in that case its cokernel would have torsion). 
Now, we would like to show that the map $\mathscr{T}_{Z} \rightarrow \mathscr{O}_{H_{w}}(-7)$ is the restriction map associated according to (13) to deletion of $H_{w}$ from the arrangement corresponding to $\tilde{Z}$, so that its kernel $\mathscr{O}_{\mathbb{P}^{2}}(-7) \oplus \mathscr{O}_{\mathbb{P}^{2}}(-4)$ will be $\mathscr{T}_{\tilde{Z}}$, thus proving the claim on freeness of $\tilde{Z}$. For this, it suffices to show that $t_{\tilde{Z}, w}=7$. We rewrite (13) as:

$$
0 \rightarrow \mathscr{T}_{\tilde{Z}} \rightarrow \mathscr{T}_{Z} \rightarrow \mathscr{O}_{H_{w}}\left(-t_{\tilde{Z}, w}\right) \rightarrow 0 .
$$

Since we have a surjection $\mathscr{T}_{Z} \rightarrow \mathscr{O}_{H_{w}}(-7)$, we must have $\left(\mathscr{T}_{Z}\right)_{\mid H_{w}} \cong \mathscr{O}_{H_{w}}(-3) \oplus \mathscr{O}_{H_{w}}(-7)$. Therefore, by the exact sequence above we deduce $t_{\tilde{Z}, w}=7$ or $t_{\tilde{Z}, w} \leq 3$. But $t_{\tilde{Z}, w} \geq 4$ since we have at least the 4 bisecant lines to $Z$ meeting at $w$ coming from $\Gamma$. The claim is now proved.

Let us now finish the proof of the theorem (still for $m=11$ ). We have several cases, according to the configuration of $\Gamma$. We give figures for the first two cases.

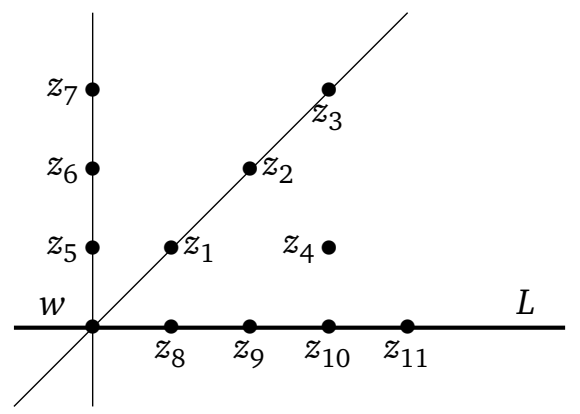

Figure 2. Case (ii)

i) $\Gamma$ consists of 2 strict 3-secants to $Z$ passing through $w$. In this case we have a point $z \in Z$ lying off the lines of $\Gamma\left(z=z_{4}\right.$ in the figure above). Since $Z$ shares the combinatorial type with a free arrangement, we have $t_{\tilde{Z}, z} \geq 5$ by Proposition 5.3. Moreover, $\tilde{Z}$ is free with exponents $(4,7)$, so in fact again Proposition 5.3 yields $t_{\tilde{Z}, z} \geq 6$. We see that this give two strict 4-secant lines to $Z$ through $z$ (although they do not show up in the picture above!). Finally we have $b_{\tilde{Z}, 5}=1$ (the line $L$ ) and $b_{\tilde{Z}, 4}=4$ (the pairs of 4 -secants through $z$ and $w$ ). This gives $b_{\tilde{Z}, 3}=9$ by (5), hence $b_{\tilde{Z}, 2}=5$ by (4). But this contradicts Hirzebruch's inequality (see [Hir83]), in the "improved" version:

$$
b_{Z, 2}+\frac{3}{4} b_{Z, 3} \geq m+\sum_{h \geq 5}(2 h-9) b_{Z, h},
$$

valid for arrangements $Z$ of $m$ complex projective lines having $b_{Z, m}=b_{Z, m-1}=$ $b_{Z, m-2}=0$.

ii) $\Gamma$ consists of 2 strict bisecant lines $L_{1}, L_{2}$ and one strict 3-secant line $L_{3}$ to $Z$ passing through $w$. Let the $\left\{z_{2 i-1}, z_{2 i}\right\}=L_{i} \cap Z$ for $i=1,2$. In this case, by the same argument as we see that, for all $i, j \in\{1,2\}$ the lines through $z_{i}$ and $z_{2+j}$ has to meet $L_{3}$ and $L$ along a point of $Z$ (although this cannot be seen in the picture above!). We get $b_{\tilde{Z}, 5}=1$ and $b_{\tilde{Z}, 4}=5$. Then $b_{\tilde{Z}, 3}=6$ by (5), hence $b_{\tilde{Z}, 2}=8$ by (4). Again this contradicts Hirzebruch's inequality. 


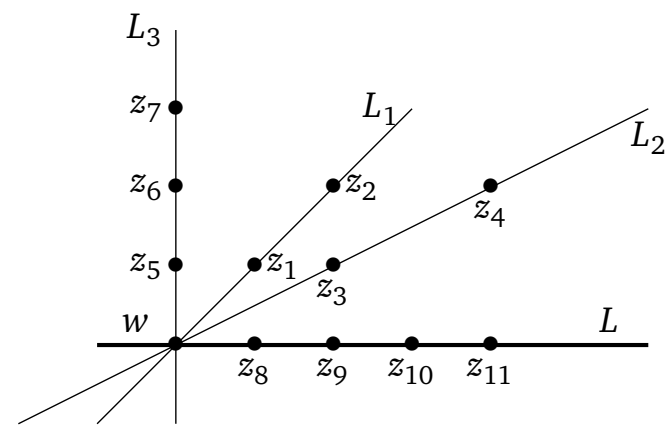

FIgURE 3. Case (iii)

iii) $\Gamma$ consists of one strict bisecant line and one strict 4-secant line to $Z$ passing through $w$. In this case we have $b_{\tilde{Z}, 5}=2$ and $b_{\tilde{Z}, 4}=0$. Using formulas (5) and (4) again we obtain a contradiction with Hirzebruch's inequality.

iv) $\Gamma$ consists of one strict 5-secant line to $Z$ passing through $w$. This case is analogous to the previous one and we omit it.

Finally, let us only sketch the idea for $m=12$. We have to deal with an arrangement $\mathscr{A}=\mathscr{A}_{Z}$ given by 12 points $Z \subset \breve{\mathbb{P}}^{2}$ having a 4-secant line $L$ and no 5 -secant lines. Again we set $Z^{\prime}=Z \cap L$ and $Z^{\prime \prime}=Z \backslash L$. By the same argument as the for the case $m=11$, we will have a point $w \in L \backslash Z$, such that all bisecant lines to $Z^{\prime \prime}$ meet $L$ either at a point of $Z$, either at $w$. We have an exact sequence analogous to (16), that provides with, this time, 6 such bisecants through $w$ (counted with multiplicity). Since $Z$ has no 5 -secant lines, this leaves as the only possibility that there are 2 strict 4-secant lines to $Z^{\prime \prime}$ through $w$, in such a way that all other bisecant to $Z^{\prime \prime}$ meets $L$ along $Z^{\prime}$. This can be proved to be impossible again by Hirzebruch's inequality, or also by a simple lemma due to Kelly, [Kel86, Lemma 2].

\section{REFERENCES}

[Dol07] Igor V. Dolgachev. Logarithmic sheaves attached to arrangements of hyperplanes. J. Math. Kyoto Univ., 47(1):35-64, 2007.

[EF80] Georges Elencwajg and Otto Forster. Bounding cohomology groups of vector bundles on $\mathbf{P}_{n}$. Math. Ann., 246(3):251-270, 1979/80.

[FMV10] Daniele Faenzi, Daniel Matei, and Jean Vallès. Hyperplane arrangements of Torelli type. ArXiv eprints, November 2010. To appear in Compositio Math.

[FV12] Daniele Faenzi and Jean Vallès. Freeness of line arrangements with many concurrent lines. In Eleventh International Conference Zaragoza-Pau on Applied Mathematics and Statistics, volume 37 of Monogr. Mat. García Galdeano, pages 133-137. Prensas Univ. Zaragoza, Zaragoza, 2012.

[Hir83] Friedrich Hirzebruch. Arrangements of lines and algebraic surfaces. In Arithmetic and geometry, Vol. II, volume 36 of Progr. Math., pages 113-140. Birkhäuser Boston, Mass., 1983.

[Kel86] Leroy M. Kelly. A resolution of the Sylvester-Gallai problem of J.-P. Serre. Discrete Comput. Geom., 1(2):101-104, 1986. 
[OSS80] Christian Okonek, Michael Schneider, and Heinz Spindler. Vector bundles on complex projective spaces, volume 3 of Progress in Mathematics. Birkhäuser Boston, Mass., 1980.

[OT92] Peter Orlik and Hiroaki Terao. Arrangements of hyperplanes, volume 300 of Grundlehren der Mathematischen Wissenschaften [Fundamental Principles of Mathematical Sciences]. Springer-Verlag, Berlin, 1992.

[Sai80] Kyoji Saito. Theory of logarithmic differential forms and logarithmic vector fields. J. Fac. Sci. Univ. Tokyo Sect. IA Math., 27(2):265-291, 1980.

[Sch00] Henry K. Schenck. A rank two vector bundle associated to a three arrangement, and its Chern polynomial. Adv. Math., 149(2):214-229, 2000.

[Ter81] Hiroaki Terao. Generalized exponents of a free arrangement of hyperplanes and Shepherd-ToddBrieskorn formula. Invent. Math., 63(1):159-179, 1981.

[Ung82] Peter Ungar. $2 N$ noncollinear points determine at least $2 N$ directions. J. Combin. Theory Ser. A, 33(3):343-347, 1982.

[WY07] Max Wakefield and Sergey Yuzvinsky. Derivations of an effective divisor on the complex projective line. Trans. Amer. Math. Soc., 359(9):4389-4403 (electronic), 2007.

[Yos04] Masahiko Yoshinaga. Characterization of a free arrangement and conjecture of Edelman and Reiner. Invent. Math., 157(2):449-454, 2004.

E-mail address: daniele.faenzi@univ-pau.fr

Université de Pau et des Pays de l’Adour, Avenue de l’Université - BP 576 - 64012 PAU Cedex - France $U R L:$ http://univ-pau.fr/ faenzi/

E-mail address: jean.valles@univ-pau.fr

Université de Pau et des Pays de l’Adour, Avenue de l’Université - BP 576 - 64012 PAU Cedex - France $U R L:$ http://web.univ-pau.fr/ jvalles/jean.html 